\title{
Los derechos humanos ante la emergencia climática
}

\author{
Human Rights in the Face of Climate Emergency
}

\author{
José Clemente Rueda Abad' \& Rocío del Carmen Vargas Castilleja²
}

\begin{abstract}
Copyright: (c) 2021
Revista Internacional de Cooperación y Desarrollo. Esta revista proporciona acceso abierto a todos sus contenidos bajo los términos de la licencia creative commons Atribución-NoComercial-SinDerivar 4.0 Internacional (CC BY-NC-ND 4.0)

Tipo de artículo: Resultado de Investigación Recibido: diciembre de 2020

Revisado: febrero de 2021

Aceptado: marzo de 2021

\section{Autores}

1 Doctor en Ciencias Sociales por la Universidad de Guanajuato. Universidad Nacional Autónoma de MéxicoPrograma de Investigación en Cambio climático. Miembro del Sistema Nacional de Investigadores Nivel 1Tutor en el Programa de Posgrado en Ciencias de la Sustentabilidad de la UNAM y en el Posgrado en Energía y Medio Ambiente de la Universidad Autónoma Metropolitana Correo electrónico: jcrueda@pincc.unam.mx ORCID: https://orcid.org/0000-0002-6207-5281
\end{abstract}

2 Doctora en Medioambiente. Facultad de Ingeniería Arturo Narro Siller-Universidad Autónoma de Tamaulipas, Centro Universitario Sur. Miembro del Sistema Nacional de Investigadores Nivel 1 y Reconocimiento como Profesor de Tiempo Completo con Perfil Deseable. Correo electrónico: rocvargas@docentes.uat.edu.mx ORCID: https://orcid.org/0000-0003-3930-6245

Cómo citar:

Rueda Abad, J. \& Vargas Castilleja, R. (2021). Los derechos humanos ante la emergencia climática. Revista Internacional de Cooperación y Desarrollo. 8(1), 95-111

DOI: $10.21500 / 23825014.4895$

G OPEN ACCESS

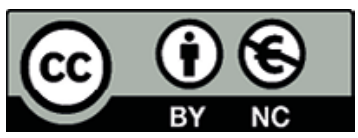

\section{Resumen}

El presente artículo tiene como objetivo mostrar de manera exploratoria la situación de los derechos humanos bajo el enfoque teórico de la metamorfosis del mundo de Ulrich Bech. En la actualidad, desde el ámbito multilateral y aportaciones de carácter académico, se reconoce que la existencia del cambio climático pone en riesgo la cumplimentación íntegra de los derechos humanos. En esta perspectiva, la teoría de la metamorfosis identifica que los derechos humanos deben transformar sus mecanismos de implementación para que sean una herramienta que cohesione una respuesta individual y colectiva ante el cambio climático. En ese sentido, se propone la implementación de una estrategia educativa de carácter transformador, que permita que las personas tengan habilidades para vivir en un mundo con una temperatura media más elevada. Desde esta lógica, al poder implementar una educación de ese tipo, estaría atendiéndose el ámbito educativo y al mismo tiempo se facilitaría la implementación de los derechos humanos.

Palabras clave: metamorfosis; cambio climático; derechos humanos; educación, reducción de riesgo.

\section{Abstract}

The present article aims at showing, in an exploratory way, the situation of human rights under the theoretical approach of the metamorphosis of the world by Ulrich Bech. At present, from the multilateral sphere and contributions of an academic nature, it is recognized that the existence of climate change puts at risk the integral fulfillment of human rights. In this perspective, the metamorphosis theory identifies that human rights must transform their implementation mechanisms so that they are a tool, which unites an individual and collective response to climate change. In this sense, the implementation of an educational strategy of a transformative nature is proposed, which allows people to have the skills to live in a world with a higher average temperature. From this logic, by being able to implement an education of this type, the educational field would be addressed, and at the same time, the implementation of human rights would be facilitated.

Keywords: Metamorphosis; Climate Change; Human Rights; Education; and Risk Reduction. 


\section{Introducción}

La idea central es que el hombre que surgirá del periodo actual de emergencia y crisis climática, requiere tener conocimientos de su entorno climáticamente cambiante al que habrá de adaptarse y en el que debe sobrevivir, y entre las cosas que debe saber y conocer, es que el Estado tiene la obligación de garantizarle sus derechos humanos.

La vinculación de los derechos humanos y el cambio climático es relativamente reciente, por lo que en el ámbito de la Convención Marco de las Naciones Unidas sobre el Cambio Climático, esta puede encontrarse por primera ocasión en Los Acuerdos de Cancún (CMNUCC, 2011, p. 2) y poco a poco ha ido tomando fuerza este tópico que debe ser fundamental en el futuro, considerando los impactos adversos probables y la posible modificación de las condiciones de la habitabilidad terrestre y humana a consecuencia del cambio climático.

Este documento está divido en tres secciones: la primera versa sobre la teoría de la metamorfosis del mundo y un enfoque humanista para la misma (Beck, 2017). En segundo lugar, se revisan los fundamentos de la emergencia climática que se deriva del anuncio del arribo a la temperatura media global al $1,5^{\circ} \mathrm{C}$ y que se traduce en la llegada del punto de retorno. Finalmente, se propone una modificación a los mecanismos de implementación de los derechos humanos a partir de la gestión integral del riesgo de desastres para que sirvan, con un enfoque cosmopolita, como herramienta de defensa de la humanidad ante el cambio climático.

Entre otras cosas, se concluye que los derechos humanos, sin mecanismos cosmopolitizados de implementación en el contexto climático, solo son vigorosos llamados desde la ética de que los gobiernos tendrían que hacer, por lo que la sola presencia de estos no servirán hasta que los ciudadanos estén conscientes de su realidad continuamente cambiante y que sepan del clima y la gestión integral del riesgo de desastres, así el pleno conocimiento de que el Estado tiene obligaciones irrenunciables en el cuidado de su integridad física y que están vinculados a la protección y salvaguarda de sus derechos humanos porque los derechos humanos con un enfoque humanista deben estar por encima de las negociaciones en el contexto climático.

\section{El enfoque humanista para la metamorfosis del mundo}

El humanismo, es una forma de entender el mundo, se trata de poner al ser humano como prioridad, es concebir a los habitantes del planeta en condiciones de equidad a pesar de los claros procesos inequitativos existentes entre países y al interior de cada uno de estos en todo el orbe.

La inequidad, es básicamente una condición estructural derivada del propio sistema económico y la construcción histórica del capital por lo que el sistema capitalista ha creado divisiones sistémicas de riqueza y pobreza que se muestran como normales (Banerjee y Duflo, 2019). Sin embargo la condición de pobreza es a todas luces una situación compleja y multidimensional que debería ser resuelta porque perse es una situación de injusticia (Sen, 2010). Situación que ha buscado ser corregida, por ejemplo, a través de programas de la agenda de la cooperación internacional instrumentada a través de agencias internacionales y programas de carácter multilateral o de algunos países para lograr que los procesos asimétricos entre los países se reduzcan, porque pensar en la desaparición de los procesos asimétricos es técnica y teóricamente imposible de conseguir.

En el contexto de cambio climático, considerando los impactos adversos potenciales que han sido compilados por el IPCC, este puede y debe concebirse como uno de los mayores retos para humanidad, tanto en marcos de carácter epistémico, como ontológico; ya que las condiciones de la habitabilidad humana habrán de modificarse y ello obliga a pensar sobre qué elementos existentes actualmente pueden ser utilizados para evitar que la inseguridad individual y colectiva genere crisis humanitarias e injusticias climáticas constantes para las generaciones del riesgo global (Beck, 2017).Uno de los re- 
tos principales en este proceso epistémico es que la sola existencia de los instrumentos (convenciones, políticas públicas, programas de gobierno, compromisos institucionales voluntarios de mitigación de gases de efecto invernadero) no son suficientes si las personas no conocen que pueden tener la capacidad de obligar a los gobiernos a hacer que dicha arquitectura institucional se cumpla para resguardar su integridad física o se busque la manera de que los derechos humanos sean violentados e incrementen su vulnerabilidad social.

Para ello, la existencia de mecanismos de garantía personal de alcance global (derechos humanos) puede ser, en un contexto de cambio climático, una herramienta para el humanismo, en ese sentido podrían articularse desde la perspectiva de la gestión integral de riesgo y una perspectiva cosmopolita. Derechos tales como la vida, la salud, la alimentación, el desplazamiento, requieren de una nueva forma de implementación porque los problemas globales que acarrea el cambio climático, en efecto tienen una dimensión mundial, pero al mismo tiempo coexisten con mecanismos nacionales para su solución que tienen que pensarse a futuro y de formas diferentes.

La teoría de la metamorfosis del mundo parte de una premisa:

El mundo no se está muriendo como creen los predicadores de catástrofes, y su rescate, como preconizan los optimistas defensores del progreso, tampoco es inminente. Antes bien, el mundo está experimentado una sorprendente pero comprensible metamorfosis mediante la transformación del horizonte referencial y de las coordenadas de acción, que tácitamente se consideran constantes e inmutables (Beck, 2017, p. 30).

La metamorfosis del mundo, a saber, contempla tres dimensiones: la metamorfosis categórica, la metamorfosis institucional y la metamorfosis político-normativa (Beck, 2017, pp. 92-93). Esta metamorfosis señala que las instituciones, las políticas públicas y la forma de entender el mundo, tal y como se pre- senta en los escenarios de futuro, no podrá ser explicado, ni entendido con las ideas que prevalecen en este momento, por ello en este planteamiento "lo más importante es cómo conceptualizar y analizar de manera empírica y contextual la continuidad y la discontinuidad, la relevancia y la sinrazón de la modernidad" (Beck, 2017, p. 88).

Esta idea de la continuidad/discontinuidad no es otra cosa que el análisis, en el sentido del realismo, de los elementos de resistencia al cambio que tienden a la reproducción social, los cuales necesaria u obligadamente deberán sufrir cambios profundos y radicales motivados y gestionados por lo que en su momento habrá de ser la realidad. Es por ello, que "la metamorfosis del mundo es algo que sucede de manera espontánea; no se trata de un programa. Metamorfosis del mundo es una expresión descriptiva, no normativa" (Beck, 2017, p. 32).

La sociedad que se está forjando y que ya vive en riesgo, tiene que buscar la "colaboración entre enemigos" (Beck, 2017, p. 60; Kahane, 2018) porque "no busca el sacrificio personal, sino el interés propio y la supervivencia" (Beck, 2017, p. 60), esta colaboración es necesaria porque "está surgiendo un nuevo horizonte global a partir de la experiencia de catástrofes pasadas y el temor a otras futuras" (Beck, 2017, p. 55).

La metamorfosis del mundo de Beck (2017) es un giro copernicano que él mismo realizó con respecto a su quehacer académico y de investigacion previa. La producción de Beck, a partir de la publicación de World risk society (Beck, 1999)' se centró en la identificación de los riesgos asimétricos en el mundo y a ello dedicó buena parte de su literatura, sin embargo en su último periodo de vida publicó tres documentos que en conjunto significan lo que el mismo Beck autodenomina como "giro copernicano 2.0"

En 2014, Beck sugiere por primera ocasión que el cambio climático puede ser el motor que ayude a las sociedades del mundo a modificar sus actividades cotidianas. En el mismo sentido, un año después identifica que los peores escenarios del cambio climático podrían servir como un catalizador que faci-

\footnotetext{
'Sociedad del resigo global.
} 
lite que la humanidad logre transitar a un nuevo estadio en el cual se logre la independencia individual y colectiva con respecto del horizonte ontológico normativo que ha causado la desestabilización del sistema climático (Beck, 2015).

Finalmente, llega a la enunciación de su modificación teórica al señalar que en el contexto de cambio climático "el mundo no gira alrededor de la nación, sino que las naciones giran alrededor de las nuevas estrellas fijas: el mundo y la humanidad" (Beck, 2017, p. 20) de acuerdo con Beck, "ello no significa, no obstante, que las naciones y los Estados nación se disuelvan y desaparezcan, sino que las naciones se metamorfosean" (Beck, 2017, p. 21) esto debe ser así porque

Es posible que la humanidad tome un camino que conduzca directamente a la autodestrucción [porque] las "certidumbres eternas" de la cosmovisión nacionalista son miopes y erróneas, por lo que pierden su obviedad en cuanto creencias de toda una época (Beck, 2017, p. 21).

El giro conceptual de Beck es redactado por primera vez en 2014 y lo confirma en 2017, y lo estructura en el siguiente orden:

El riesgo global tiene dos caras: la traumática vulnerabilidad de todos y la consiguiente responsabilidad de todos, incluida la propia sobrevivencia. Nos obliga a recordar la propia supervivencia. Nos obliga a recordar las distintas maneras en que la raza humana pone en peligro su propia existencia. La conciencia de humanidad actúa como un punto fijo [...] Quien habla acerca de la humanidad no está haciendo trampas [...] sino que se ve obligado a salvar a los demás para salvarse a sí mismo (Beck; 2017, p. 60).

Esta visión del interés individual y colectivo, ser víctimas y victimarios y al mismo tiempo una responsabilidad compartida derivada de los impactos probables del cambio climático sin tener como premisa la soberanía nacional hace que Beck, en esta última fase de su producción intelectual, pueda insertarse en el ámbito de la ecosofía.

La ecosofía "ha subrayado desde sus comienzos que para mitigar o superar los aspectos negativos de tal desorden material habrá que tener en cuenta la raíz ideológica y espiritual de la problemática, síntoma de un cambio histórico sin precedentes: el hecho de que la especie humana haya devenido una variable física en el sistema físico del planeta (Bugallo, 2010, p. 152) se trata de una dimensión nueva en el horizonte ontológico normativo preexistente entre la relación humanos y medioambiente. Un ejemplo de este nuevo tipo de pensamiento es la Carta de la Tierra ya que en ella se muestra "la comprensión universalmente compartida sobre la interdependencia entre los seres humanos y la naturaleza" (Comins Mingol, 2016, p. 145).

En otras palabras "lo importante a destacar para el planteamiento ecosófico, es que si la consciencia impregna todo ser, entraña una nueva forma de ver al ser humano no como individuo, sino como persona, es decir, como un nudo de relaciones (Sepúlveda Pizarro, 2018, p. 267). Es lo que Beck denomina como la doble cara del riesgo global y la necesidad de colaborar entre todos, para que todos se salven.

En términos de Tamayo Pérez (2017), "si la humanidad pretende contar con un futuro requiere curarse de la locura ecocida, esa egosintónica, thanática e ignorante que tan nefastos efectos produce a todos los ecosistemas del planeta" (p. 52) pero para ello requiere, en principio, entender que es parte de la problemática y por el solo hecho de pensar como el sistema nos ha enseñado a pensar y recrear las estructuras de dominación en ese entendido, como señala Beck, se requiere una metamorfosis basada en su denominado giro copernicano.

Entre los elementos que habrán de ayudar a la metamorfosis del mundo se encuentran los derechos humanos. De acuerdo con Beck (2017), "los derechos humanos hacen que las jerarquías globales, que los colonizadores percibían como 'bondades naturales' se transformen en 'males políticos' que transgreden el orden normativo del mundo" (p. 75). 
En ese sentido, desde la óptica de la teoría de la metamorfosis del mundo, el problema de los derechos humanos se encuentra, no en su denominación o categorización, sino en su implementación ya que como política pública siguen apuntando al ámbito del nacionalismo metodológico (Beck, 2017, p. 20) y no hacia el cosmopolitanismo global.

Por ello, el orden social y sus instituciones deben ajustarse a las futuras realidades que habrá de imponer el cambio climático en todo el orbe, por lo que debe ponderarse la modificación institucional de la gobernanza ambiental (Schwarz-Herion, 2018; Dryzek, 2016; Gardiner, 2011; Meadowcroft, 2009; Ostrom, 2009; Young, 2013; Paavola, 2007; Paavola, 2008; Biermann \& Pattberg, 2012) por una que incorpore elementos de carácter económico (Tol, 2015; Helm, 2016; Nordhaus, 2014; Nordhaus, 2015) que sirva para cumplir con los objetivos del Acuerdo de París (Bodansky, 2016; Keohane \& Oppenheimer, 2016; Stavins, 2016a; Stavins, 2016b) y la Agenda 2030 de los Objetivos para el Desarrollo Sostenible (Hou, Walsh \& Zhang, 2015; de Loma-Osorio, 2016; Hák, Svatava y Moldan, 2015; Sánchez Rodríguez, Urge-Vorsatz \& Salisu, 2018) lo cual supondría -cuando menos hipotéticamente-que se podría reducir el potencial de riesgo de desastres que se visualizan en los escenarios climáticos (Trogrlić et al., 2017; Weichselgasrtner \& Pigeon, 2015; Pearson \& Pelling, 2015). Dicho en otros términos, intentar hacer algo como lo antes mencionado podría ser una hoja de ruta que modifique los escenarios climáticos que se ciernen sobre la humanidad. Lograrlo, sería un avance en la reducción de las desigualdades.

Esta vinculación es necesaria porque:

La justicia climática solo puede ser una realidad si se logran los ODS, al igual que los objetivos climáticos. Los ODS contienen las condiciones necesarias que deben cumplirse a nivel nacional e internacional, además del cumplimiento de los compromisos reflejados en las NDC y en el Acuerdo de París (de Loma-Osorio, 2017 p. 4).

Sin embargo, desde el punto de vista de la teoría de la metamorfosis del mundo "la cosmopoli- tización crea horizontes normativos de igualdad y justicia, ejerciendo presión para que se produzca así un cambio inclusivo en las actuales estructuras e instituciones de la desigualdad y el poder global (Beck, 2017, p. 75).

\section{Fundamentos de la Emergencia Climática}

La emergencia climática, de acuerdo con el Oxford Dictionaries, puede ser definida como "una situación en la que se requieren acciones urgentes para reducir o detener el cambio climático y evitar daños ambientales potencialmente irreversibles como resultado de él" (Oxford University Press, 2019). De acuerdo con el mencionado diccionario, este término ha sido el más usado en el contexto del discurso climático durante el año 2019.

Pareciera que esta sensación de emergencia es nueva, sin embargo, es oportuno mencionar que la sensación de urgencia fue incorporada en el discurso de las Naciones Unidas, desde una década antes cuando, el 3 de septiembre de 2009, Ban Ki-Moon -en la Tercera Conferencia Mundial del Clima, celebrada en la sede de la Organización Meteorológica Mundial (OMM, en adelante) en el segmento de Alto Nivel-, le dijo a los Jefes de Estado ahí presentes: "Hemos desatado fuerzas poderosas e impredecibles cuyo impacto ya es muy visible. Lo he visto por mí mismo [...] Déjenme decir claramente: sabemos cuál es el problema. Sabemos lo que debemos hacer. Ahora es el momento de hacerlo. Ahora es nuestro momento" (Ki-Moon, 2009).

La noción de emergencia climática se vincula de manera directa a los datos científicos más recientes que han sido publicados tanto por el IPCC y la OMM, así como por los resultados de la Conferencia de las Partes 24 (COP, en adelante) (celebrada en 2018 en Katowice, Polonia) y que obligó a la reaparición de la Coalición por la Gran Ambición, que fue quien hizo el primer llamado para que los países incrementaran sus Contribuciones Nacionalmente Determinadas (CND), en el sentido que recomienda el IPCC (HAC, Dec 14, 2018). 
Todo esto tiene una historia: previo a la COP21, la Convención Marco de las Naciones Unidas sobre el Cambio Climático, públicó un estudio con las CND que habían llegado dentro del límite de tiempo establecido para tal efecto, y concluyó que con la suma global de todas las CND que recibió, el esfuerzo de mitigación a realizar por el conjunto de países, no sería posible garantizar la reducción de emisiones suficientes para lograr los $2^{\circ} \mathrm{C}$ y menos el $1,5^{\circ} \mathrm{C}$ (CMNUCC, 2015b), con dicho resultado la COP21 concluyó con el anuncio del Acuerdo de París de apostar por los mencionados $2^{\circ} \mathrm{C}$ y que de ser posible (ampliar la ambición) por el $1,5^{\circ} \mathrm{C}$ y en consecuencia, pidió al IPCC la elaboración de un reporte especial para determinar la factibilidad científica de la decisión política tomada por la COP en París (CMNUCC, 2015a).

El reporte especial solicitado al IPCC fue presentado al mundo en octubre de 2018 y, en términos generales dice que el mundo habrá de llegar al $1.5^{\circ} \mathrm{C}$ en el 2040 y a los $2^{\circ} \mathrm{C}$ en el 2060 , por ello ha dicho que el punto de no retorno para la humanidad está colocado antes de la mitad del siglo XXI. Para evitar que esto suceda se tendrían que implementar cambios a escala planetaria que van más allá de la simple modificación de la demanda y consumo de energía, se tendría que hacer un conjunto de cambios a gran escala para poder aspirar a tener emisiones cero en el 2050 y a partir de dicho momento emisiones negativas (IPCC, 2019).

Sin embargo, es importante destacar que en el periodo 2013-2014 fue presentado el Quinto Reporte de Evaluación del IPCC en el que se señala que la información disponible en ese momento no hacía suponer que los $2^{\circ} \mathrm{C}$ fueran posible (IPCC, 2014a, p. 16).

En el mismo sentido en la primera semana de diciembre de 2019 se ha presentado el Informe sobre la disparidad en las emisiones de 2019 del Programa de las Naciones Unidas para el Medio Ambiente, que señala que considerando el ritmo actual de reducción de emisiones lo que se podría esperar hacia finales del presente siglo son temperaturas medias globales entre los 3 y los $4^{\circ} \mathrm{C}$ (PNUMA, 2019).
Considerando lo antes mencionado, es importante señalar que el balance quinquenal del estado del clima de la OMM correspondiente al periodo 2015-2019, señala un aumento de $20 \%$ en las emisiones de gases de efecto invernadero, un aumento de $0.2^{\circ} \mathrm{C}$ más sobre el periodo 2010-2015y que los indicadores del sistema climático se siguen alterando de manera negativa y el número de olas de calor han aumentado y se han presentado en todas las masas continentales (WMO, 2019).

En lo que respecta a la elevación de la temperatura, el Fifth Assessment Report (AR5) señala que si no se realiza una mitigación adicional se experimentarán incrementos en la temperatura media global en 2100 de $3.7^{\circ} \mathrm{C}$ a $4.8^{\circ} \mathrm{C}$ en comparación con los niveles preindustriales (IPCC, 2014a, p. 8), razón por la cual, este cambio climático puede comenzar a verse como irreversible en una escala temporal de entre varios siglos y milenios (IPCC, 2013 p. 28).

Los escenarios de impactos que documenta el IPCC confirman la "democratización del riesgo" ya que desde la lógica argumentativa que manejan, lo que se infiere es que no hay sectores o regiones geográficas del planeta que vayan a quedar indemnes de los impactos adversos del cambio climático y lo otro que debe considerarse, es que al hablar de estabilizar la temperatura en $2^{\circ} \mathrm{C}$ o menos, significa que el cambio climático es algo que ya no puede revertirse, y por ello es mejor que se trabaje por lograr los $2^{\circ} \mathrm{C}$, a tener $4^{\circ} \mathrm{C}$ o $5^{\circ} \mathrm{C}$ más en la temperatura media global hacia finales del presente siglo.

Todo lo visto hasta el momento, no adquiere sentido social, sino se coloca ahí a las personas. En ese contexto, el cambio climático se debe comprender como un riesgo estructural (Mancini, 2018, p. 18); en otras palabras, la seguridad humana ante el cambio climático está comprometida porque las estructuras políticas no habrán de modificarse en el ritmo e intensidad que el problema demanda, lo cual pone en riesgo el sentido humanista que debieran tener los gobiernos en el cuidado y protección de los habitantes de sus territorios nacionales. 
De acuerdo con el IPCC, la seguridad humana se enfrentará a diferentes escenarios como fenómenos migratorios, la pobreza y las crisis económicas pueden generar un incremento en los conflictos violentos, y los impactos en la infraestructura esencial pueden gestar problemas a la seguridad nacional, inseguridad alimentaria y hambrunas, e incremento de la pobreza en zonas urbanas (IPCC, 2014b, p. 20).

En la sentencia de Beck, de reconocer que todos somos vulnerables y todos somos responsables, no está diciendo que todos seamos responsables de las causas del problema, sino que todos somos responsables de cambiar nuestras conductas y de asumir que el cambio climático nos hace asimétricamente vulnerables por ello debe hacernos cambiar para que la metamorfosis colectiva pueda ser una realidad.

En ese sentido, Ivanova y Wood (2020) en un estudio realizado en la Unión Europea encuentran que el percentil superior de emisores es responsable del $27 \%$ de las emisiones, y el $1 \%$ superior de los emisores supera las emisiones anuales per cápita de $55 \mathrm{t}$ $\mathrm{CO}_{2}$-eq. Por su parte, Gössling y Humpe (2020) señalan que en el caso de la aviación sus datos sugieren que "el percentil de los viajeros más frecuentes, como máximo el 1\% de la población mundial, probablemente representa más de la mitad de las emisiones totales de los viajes aéreos de pasajeros" (p.1)

En el periodo 1988-2015 las emisiones acumuladas por 100 empresas contabilizan el 70,57\% de las emisiones globales. De ese total global de empresas, 32 son estatales y han contribuido con $39,25 \%$ de dichas emisiones; 66 empresas son carácter privado y han contribuido con el $30,9 \%$ y solo 2 empresas de carácter mixto, contribuyeron con 0,42\%. La lista completa que no aparece en el reporte Carbon Majors Report 2017 (Criffin, 2017) fue publicada por el periódico británico The Guardian (2017) y sigue estando disponible para su consulta.

Revisando los primeros 50 lugares, que aparecen en el Carbon Majors Report 2017 (Griffin, 2017, p. 14) estos representan el $63,18 \%$ de las emisiones en tanto que las restante 50 empresas solo contri- buyen con el 7,39\%, en ese escenario, en el top 50, 23 empresas estatales son responsables del 37,56\% del total emitido en el periodo, por su parte $27 \mathrm{em}$ presas privadas fueron responsables del $25,62 \%$. En este caso, las empresas analizadas forman parte del sector energético, "agravan la crisis climática y [amenazan] el disfrute efectivo de los derechos humanos" (Albar et al., 2020, p. 7).

Otro de los elementos que atentan contra la humanidad, es que el sector energético convencional y extractivista sigue accediendo a fuentes de financiamiento de carácter privado. De acuerdo con el Reporte Banking on Climate Change. Fossil Fuel Finance Report 2020, se señala que

Desde la adopción del Acuerdo de París a fines de 2015, los 35 bancos en el alcance de este informe han otorgado US $\$ 2,7$ billones en préstamos y suscripción a la industria de combustibles fósiles, con un aumento anual de financiamiento fósil cada año. JPMorgan Chase se convirtió en el primer banco en superar la marca de un cuarto de billón de dólares en financiamiento fósil posterior a París, con \$269 mil millones en 2016-2019 (Kirsch et al., 2020, p. 4).

En otras palabras, el proceso de descarbonización económica que manda el régimen climático internacional está en riesgo no solo porque se continua invirtiendo en el sector de combustibles fósiles, sino porque la inversión en el sector de energías alternativas no garantiza que se logre la modificación de la matriz energética mundial antes de que la elevación de la temperatura llegue al punto de no retorno.

\section{Los derechos humanos como mecanismo de res- guardo humanista ante la emergencia climática}

En el momento actual, los datos de los reportes quinquenales sobre los impactos ya registrados en la última década por la Organización Meteorológica Mundial (WMO, 2016; WMO, 2019), además de los datos del Índice de Riesgo Climático del Cerman Watch Institute (Eckstein, Kunzel \& Schafer, 2018) y el 
análisis de riesgo global del Foro Económico Mundial (World Economic Forum, 2019, p. 5 y 7), en conjunto, muestran que la integridad de las personas y todos sus derechos humanos no podrán ser garantizados totalmente por los Estados nacionales por lo que en la práctica se requiere de un cambio de implementación para hacer que las personas puedan estar protegidas.

El trabajo que ha realizado la ONU a través del Consejo de Derechos Humanos, desde el 2008, puede ser revisado en sus resoluciones, ${ }^{2}$ sin embargo, estas solo son "vigorosos pronunciamientos éticos sobre lo que se debe [...] hacer. [...] e indican que algo tiene que hacerse para la realización de esas libertades reconocidas que se han identificado a través de estos derechos" (Sen, 2010, p. 389-390).

En el ámbito de la investigación, puede afirmarse que la vinculación y análisis de los derechos humanos y el cambio climático son relativamente recientes y por ello es un área de frontera académica. Entre algunos de los tópicos que se han comenzado a estudiar se encuentra la vinculación de estas dos áreas con el desarrollo sustentable (Boyle, 2020) la gobernanza (Koivurova, Duyck \& Heinämäki, 2013; UNEP \& Columbia Law School, 2015), la instrumentación de estos derechos (Caney, 2008; CIEL \& FES, 2009; CIEL \& CARE, 2015; Schapper \& Lederer, 2014; Krämer, 2019), la protección de los derechos de los niños (Arts, 2019), la justicia climática (Toussaint \& Martínez, 2019; Derman, 2020), la necesidad de que se cumplan los propósitos del Acuerdo de París (Blau, 2017) y sus instrumentos, específicamente las Contribuciones Nacionalmente Determinadas (Clemarec, 2019), así como discusiones sobre el modelo de derechos humanos que se encuentra en crisis por el cambio climático (Behnassi, 2019). Es importante señalar que en el Acuerdo de París la cuestión de los derechos humanos se encuentra en la parte del preámbulo, porque se considera que es un principio, que ya está consagrado en otros instrumentos multilaterales que han sido firmados por muchos de los

\footnotetext{
${ }^{2}$ Resolución 42/21 de julio de 2019, Resolución 38/ de julio de 2018, Resolución 35/20 de julio de 2017, Resolución 32/33 de julio de 2016, Resolución 29/15 de julio de 2015, Resolución 26/27 de julio de 2014, Resolución 18/22 de septiembre de 2011, Resolución 10/4 de marzo de 2009) y la Resolución 7/23 de marzo de 2008 (OHCHR, 2020)
}

países que han ratificado el Acuerdo climático y la obligatoriedad de la cumplimentación se encuentra en los documentos relacionados con los derechos humanos (Duyck, et al., 2017).

Los derechos humanos que serán puestos en riesgo por el cambio climático son: el derecho a la vida digna, a la salud, al agua, a un medio ambiente sano, a una alimentación y a una vivienda adecuada (Carmona Tinoco, 2016, pp.19-27) al que habría que agregar el derecho al desplazamiento social. Por lo que desde la doxa actual, los Estados tienen la obligación de dar las garantías a sus ciudadanos para que estos derechos no sean violentados y en el contexto de cambio climático, esto iniciaría con la implementación de acciones normativas y la instrumentación de políticas públicas enfocadas tanto a la mitigación de GEI como a la adaptación social.

A manera de ejemplo, en el caso específico de las migraciones asociadas al clima, es necesario decir que existe un debate sobre la atribución del cambio climático como factor en el desplazamiento de las personas (Lonergan \& Swain, 1999; Hsiang \& Sobel, 2016; Bedarff \& Jakobeit, 2017), así como la coexistencia de un debate académico sobre si a estos migrantes se les puede denominar como refugiados ambientales (El-Hinnawi, 1985; Black, 2001) o refugiados climáticos (Farbotko \& Lazrus, 2012; Methmann \& Oels, 2015; Baldwin, 2020). En efecto, puede afirmarse que como área de investigación se han logrado avances, pero en el lado práctico sigue presentando retos para la implementación.

En el ámbito regional, el cambio climático "influye, al parecer, en la movilidad humana en Centroamérica y el Caribe, aunque aislar los impulsores ambientales de los otros factores de la migración sigue siendo complejo" (Organización Internacional para las Migraciones, 2019, p. 112). Incluso, en un análisis regional realizado en el Sahara, Asia Meridional y América Latina hacia mediados del presente siglo se proyecta que al menos 143 millones de personas serian desplazados internos por causas climáticas (Kumari Rigaud, et al., 2018).

Desde el ámbito institucional, la región ha gestado procesos de discusión y ha realizado aportacio- 
nes para el diseño del Pacto Global para la Migración Segura, Ordenada y Regular (Canales \& Rojas, 2017). En lo que se refiere a las políticas públicas y en el contexto de los instrumentos del Acuerdo de París, las Contribuciones Nacionalmente Determinadas y las Comunicaciones Nacionales de los países de la zona, existe el reconocimiento del cambio climático y su vinculación con la cuestión migratoria, sin embargo "a nivel general, se observa una escasa consolidación de avances en la integración de la movilidad humana en las estrategias nacionales de cambio climático" (Lejtreger, 2018, p. 54). Además de ello, existe un plan de alcance regional que se conforma por Costa Rica, El Salvador, Guatemala, Honduras, Nicaragua y Panamá, que es fruto de la Declaración de San Pedro Sula del año 2008 (Comisión Centroamericana de Ambiente y Desarrollo, 2010) y que ha sido recientemente actualizado para estar vigente en el periodo 2018-2022 (Comisión Centroamericana de Ambiente y Desarrollo, 2018) y en donde se considera la vinculación del cambio climático y la migración, pero no es el centro de las problemáticas sociales del fenómeno.

Aunque existen estudios diversos sobre la región centroamericana y México (Vega, 2005; Carr, 2008; Alscher, 2010; Albo \& Ordaz, 2011; Conzález, Salazar \& Cruz, 2012; Nawrotzki, Riosmena \& Hunter, 2013; Lozano Sivisaca, Chacón-Cascante, Gutiérrez Montes \& Robalino, 2015; Corona \& Ortiz, 2015, 2019; Aragonés y Salgado, 2015; Ochoa Lupián \& Ayvar, 2015; Corona, 2018; Canales, Fuentes \& Rosa de León, 2019) lo cierto es que "los pocos indicios sobre una posible vinculación relacional entre los cambios climáticos y la migración centroamericana de paso por México invitan a realizar investigaciones empíricas sobre el tema (Casillas, 2020, p. 90).

La salvaguarda de los derechos humanos en el contexto climático requiere que los Estados tengan legislaciones específicas y otras de carácter temático que puedan vincularse a los derechos que están en riesgo (cambio climático, mitigación de gases de efecto invernadero, adaptación social al cambio climático, educación, sensibilización y comunicación del cambio climático, salud, agua, medio ambiente sano, alimentación adecuada y a la vivienda), ade- más deben existir planes y programas de gobierno tanto federales como subnacionales en dichos temas. En el mismo sentido, se requiere que los países hayan firmado y ratificado instrumentos de carácter multilateral como son la Convención Marco de las Naciones Unidas sobre el Cambio Climático, el Acuerdo de París y la entrega e instrumentación de la Contribución Nacionalmente Determinada, que en el caso de los países latinoamericanos casi todos cuentan con dichos instrumentos.

Si lo antes enunciado no existe en un ente nacional, entonces ese gobierno estará violando los derechos humanos de sus ciudadanos. En otras palabras, la violación de los derechos humanos no se da entre conciudadanos, sino que es el Estado el que a través de sus acciones, inacciones o mala implementación desde el gobierno vulnera los derechos consagrados a sus ciudadanos. El reclamo de una violación a los derechos humanos se ejerce a partir de una demanda de parte de un ciudadano, o un colectivo de ciudadanos, ante la instancia correspondiente, que deberá darle seguimiento, si la causa está fundada, se investiga y en caso de ser procedente se estaría elaborando una recomendación al gobierno para que resarza el daño; es decir, que se corrija la violación de los derechos que un ciudadano ha demandado se le respeten. Es importante señalar que la Comisión Interamericana de Derechos Humanos ha abierto una Relatoría Especial sobre Derechos Económicos, Sociales, Culturales y Ambientales que puede servir como un espacio para la defensa del medioambiente sobre todo cuando las instancias nacionales se agotan (Auz, 2018).

Una alternativa sería vincular el cambio climático con la gestión integral de riesgo de desastres, ${ }^{3}$ lo cual abre una ruta al entendimiento del problema y los impactos que se prospectan desde la literatura científica que habrán de ocurrir en el corto, mediano y largo plazo. Lo que se traduce a un hecho: los ciudadanos deben ser conscientes del futuro que les espera y en casos específicos saber que hacer no solo

\footnotetext{
Puede entenderse como "un proceso social cuyo fin último es la previsión, la reducción y el control permanente de los factores de riesgo de desastre en la sociedad, integrada y en consonancia, con el logro de pautas de desarrollo humano, económico, ambiental y territorial, sostenibles" (Narváez, Lavell \& Pérez, 2009, p. 33)
} 
en el ámbito individual, sino que además deben conocer cuáles son las tareas que debe ejecutar el gobierno. Para ello, los actores deberán:

a) Identificar el riesgo mediante su análisis y evaluación, teniendo los derechos humanos (DESCA) como eje articulador de dicha identificación y reconociendo que tales derechos deben ser la base de los diagnósticos correspondientes.

b) Reducir el riesgo mediante las acciones de prevención y mitigación de daños, diferenciando los aspectos vinculados con los derechos humanos de los relacionados con infraestructura y sectores económicos.

c) Manejar y operar las acciones de respuesta y recuperación en los términos igualmente de introducir el enfoque de los derechos humanos, procurando que la intervención del sector privado empresarial se apegue al interés público más que al lucro especulativo.

d) Establecer lineamientos de política para aplicar la transferencia financiera del riesgo, el cual supone implementar mecanismos de protección financiera para solventar los riesgos y daños o pérdidas y la mitigación o reducción de emisión de gases de efecto invernadero (CEI); en este campo es preciso definir criterios para fortalecer la prevención desastres y la adaptación preventiva frente a cambio climático (CNDH, 2018, p. 61).

Para lograr esto se requieren, al menos, dos elementos: generar una institución autónoma en la administración pública que facilite y apoye la elaboración de demandas ciudadanas en el ámbito climático y, en segundo lugar, capacitar y educar a los ciudadanos de ahora y del futuro en materia de desastres.

Como se ha mencionado, la implementación actual de los derechos humanos requiere que exista un Observatorio ciudadano que tenga la capacidad legal para realizar demandas ante la entidad gubernamental encargada de hacer que los derechos humanos se respeten.
En el segundo elemento, de acuerdo con López Morales (2019) hay que considerar que este enfoque educativo debe moverse en el supuesto de que "para enfrentar cualquier situación que nos pone en peligro, primero debemos conocerla, saber por qué pasa y de qué manera podemos adaptarnos para reducir el riesgo ante un desastre" (p. 178).

Esta parte educativa puede sustentarse en el marco de los derechos humanos, porque como señala la Organización de las Naciones Unidas para Educación, la Ciencia y la Cultura "urge responder al cambio climático con políticas eficaces e integrales, que respeten y promuevan los derechos humanos y se guíen por principios éticos" (Unesco, 2017, p. 162).

El centro del debate en esta discusión debe ser: ¿qué tipo de habilidades debe tener para poder sobrevivir en un mundo cambiante? En ese sentido las nuevas generaciones deben ser educadas o capacitadas para tener habilidades específicas para un mundo con un clima diferente al actual, por ello:

Al pensar en un panorama con 203 grados más, a todo mundo y en especial iniciando desde niños, se les debe enseñar habilidades enfocadas a la adaptación y mitigación de estos efectos, producción de alimentos, gestión de los recursos disponibles, captación y gestión del agua, materiales y sistemas constructivos adaptables a esas variaciones, innovación y desarrollo hacia la producción y comercialización de bienes y servicios; buscando el cambio del paradigma que provoca el cambio climático (Castañeda, 2020).

Si a las nuevas generaciones se les comienza a educar de una forma diferente, donde se tenga claro que el Estado no podrá garantizarle nada y que las respuestas no necesariamente vendrán de una figura de autoridad sino de la aplicación de un razonamiento cosmopolita que le diga que la solidaridad y la colaboración no solo lo harán más humano, sino que la estrategia de respuesta se encuentra en una visión amplia que une a la humanidad en la posibilidad de un conjunto de desastres en los que la evaluación individual de los riesgos comunes puede no 
solo ayudarles de manera individual sino colectiva, para continuar existiendo.

\section{Conclusiones}

Se ha fundamentado la necesidad de considerar la teoría de la metamorfosis y la emergencia climática para iniciar la transformación del mundo con una ciudadanía conocedora y consiente de su derecho a un entorno digno y resiliente ante los efectos adversos del cambio climático.

Se requiere un cambio obligado y categórico con visión global con el objeto de crear una alianza sólida y efectiva para estar preparados para las condiciones futuras. Las instituciones deben implementar prácticas no antes vistas, pues el proceso de transformación es inminente y urgente. La integridad física de las personas está en manos del Estado, sin embargo, su composición actual y su desordenada ejecución, no permite ni permitirá, preservar los derechos humanos.

Los esfuerzos de divulgación, conformación de evidencias e informes del IPCC, WMO, Unesco, PNU$M A$, entre otros entes internacionales y articuladores de los derechos humanos, han puesto en total evidencia la urgente necesidad de atender el Ilamado, por lo que el Estado mismo, tiene la responsabilidad de actuar para resguardar al ser humano y en contraparte, la ciudadanía debe tener la voluntad de repensar, comprender, aprender y reflexionar profundamente sobre la huella que está dejando a su paso, pero trabajando bajo un enfoque, climáticamente, responsable.

La existencia del cambio climático y sus escenarios, deben obligar a la humanidad a pensar y a hacer las cosas de una manera diferente; porque el hecho de saber que el riesgo climático existe es una forma de tomar conciencia sobre lo que nos depara el futuro, y en efecto ya hay algunos grupos sociales, como los jóvenes de diversos lugares del mundo, que comienzan a demandar acciones concretas a sus gobiernos, aunque muchas veces es el sector privado el que debería ser llamado a modificar sus acciones, como es el caso de la emisiones globales acumuladas en el periodo 1988-2015 que amenazan el disfrute efectivo de los derechos humanos.

Desde la postura teórica asumida, no se desconocen los avances institucionales en materia de derechos humanos, los cuales oscilan en escalas que van de lo subnacional a lo global, sin embargo, sus mecanismos de implementación son insuficientes, y en un futuro cercano serán disfuncionales porque siguen ejecutándose en escala de lo nacional algo que es de alcance global. Ante esta circunstancia, aunque Beck no lo menciona en estos términos, la consagración de los derechos humanos es el caballo de Troya que el sistema normativo imperante se ha colocado.

Pensando en un futuro climáticamente diferente al actual, el reto de la educación de las nuevas generaciones puede convertirse en el lugar donde se gesten áreas de cooperación, no solo entre académicos, investigadores y pedagogos para diseñar mapas curriculares que no solo den a conocer el problema, sino que doten a los estudiantes de las herramientas necesarias para sobrevivir y conocer sus derechos humanos. En un plano más amplio, si las nuevas generaciones aprenden a cosechar agua de lluvia, cosechar en huertos urbanos, apropiarsen socialmente de la energía, entre muchas cosas, ayudaría a garantizar los derechos humanos porque se les estaría dotando de los conocimientos apropiados para no depender, en todo momento, del Estado. 


\section{Referencias}

Albar Díaz, M., Auz, J., Bautista López, J., Bustos, C., Carballo, J. M., Castillo Barnetche, V., Gumucio, C., Lavayen, A., Martínez, A., Medici-Colombo, G., Pineda, C. \& Tan, J. (2020). Cambio Climático y los Derechos de Mujeres, Pueblos Indígenas y Comunidades Rurales en las Américas. Bogotá, Colombia. Fundación Heinrich Böll, Oficina Bogotá - Colombia, Alianza Hondureña ante el Cambio Climático, AIDA, CEDAT, CELS, CEMDA, Conectas, Dejusticia, DPLF, Earthrights International, Engajamundo, FARN, FIMA, Fundación Pachamama, FUNDEPS, IDL y La Ruta del Clima. https://mx.boell. org/sites/default/files/2020-05/hbs_Cambio_climatico\%20en\%20las\%20Americas_web_o.pdf

Albo, A. \& Ordaz, J. L. (2011). Migración y Cambio Climático. El caso mexicano, Documento de Trabajo Número 11/27, México, BBVA Research Mexico, Servicio de estudios económicos del grupo BBVA.

Alscher, S. (2010). Environmental factors in mexican migration: The cases of Chiapas and Tlaxcala. Environment, Forced migration and social vulnerability, 171-185.

Aragonés, A. M. \& Salgado, U. (2015). Cambio climático y migración: Zacatecas y San Luis Potosí, un estudio de caso. En Ana María Aragonés (Coord.). Consecuencias del cambio climático sobre la migración: un análisis para México.. México: UNAM-Instituto de Investigaciones Económicas. pp. 167-194

Arts, K. (2019). Children's Rights and Climate Change. In Children's Rights and Sustainable Development: Interpreting the UNCRC for Future Cenerations / Edited by Claire Fenton-Clynn (Series: Treaty Implementation for Sustainable Development). pp. 216-235. doi:10.1017/9781108140348.010

Auz, J. (2018). ¿Por qué el Sistema Interamericano de Derechos Humanos se rezaga en materia de cambio climático? https://aida-americas.org/es/prensa/ organizaciones-alertan-ante-la-cidh-de-impactos-de-la-crisis-climatica-en-derechos-humanos

Baldwin, W. A. (2020). Climate Change Refugees. International Encyclopedia of Human Ceography, 2da. edition, Volume 2. Elsevier. DOI: https://doi. org/10.1016/B978-0-08-102295-5.11000-5

Banerjee, A. V. \& Duflo, E. (2019). Repensar la pobreza. Un giro radical en la lucha contra la desigualdad global. México, Taurus

Beck, U. (1999). World risk society. Cambridge, Polity, 1999.
Beck, U. (2014). How climate change might save the world: metamorphosis. Harvard Desing Magazine, 39, pp.88-98

Beck, U. (2015). Emancipatory catastrophism: What does it mean to Climate change and risk society? Current sociology, 63(1), 75-88.

Beck, U. (2017). La metamorfosis del mundo. España, Paidós Col. Estado y Sociedad, 249p.

Bedarff, H. \& Jakobeit, C. (2017). Climate Change, Migration, and Displacement. The Underestimated Disaster, 38p.

Behnassi M. (2019). Mainstreaming a Rights-Based Approach in the Clobal Climate Regime. In Behnassi, M., Gupta, H. \& Pollmann, O. (Eds). Human and Environmental Security in the Era of Clobal Risks. Springer, Cham. DOI https://doi. org/10.1007/978-3-319-92828-9_1

Biermann, F. \& Pattberg, P. (Eds.). (2012). Clobal Environmental Covernance Reconsidered, Massachusetts Institute of Technology.

Black, R. (2001). Environmental refugees: Myth or reality? Working Paper 34, Ginebra: United Nations High Commissioner for Refugees (UNHCR).

Blau, J. (2017). The Paris Agreement: climate change, solidarity and Human Rights. New York: Palgrave Macmillan, 119p. ISBN 9783319535401

Bodansky, D. (2016). The Paris Climate Change Agreement: a new hope? American Journal of International Law, 110. http://papers.ssrn.com/sol3/ papers.cfm?abstract_id $=2773895$

Boyle, A. (2020). Climate Change, Sustainable Development, and Human Rights. En, Markus Kaltenborn, Markus Krajewski \& Heike Kuhn (Eds.). Sustainable Development Goals and Human Rights. Switzerland, Springer Nature, Interdisciplinary Studies in Human Rights Volume 5, 239p. pp.171-190. DOI: https://doi.org/10.1007/978-3030-30469-0

Bugallo, A. (2010). 'Filosofía ambiental; nuevo pensamiento sobre ecosofía práctica y diversidad biocultural'. En Scannone Juan Carlos S. J. (ed.), Un nuevo pensamiento para otro mundo posible, Universidad Católica de Córdoba, Córdoba, Argentina, pp. 151-174, ISBN 978-987-626-1081. 
Canales, A. \& Rojas, M. L. (2017). Panorama de la migración internacional en México y Centroamérica. Documento elaborado en el marco de la Reunión Regional Latinoamericana y Caribeña de Expertas y Expertos en Migración Internacional preparatoria del Pacto Mundial para una Migración Segura, Ordenada y Regular, Santiago de Chile: CEPAL.

Canales, A., Fuentes, J. A., \& Rosa de León, C. (2019). Desarrollo y migración: desafíos y oportunidades en los países del norte de Centroamérica (LC/MEX/ TS.2019/7), Ciudad de México: Comisión Económica para América Latina y el Caribe (CEPAL).

Caney, S. (2008). Human rights, climate change, and discounting. Environmental Politics, 17(4), 536-555. DOI:10.1080/09644010802193401

Carmona Tinoco, J. U. (Coord.). (2016). Cambio climático y derechos humanos. México, Comisión Nacional de los Derechos Humanos, 39p.

Carr, D. (2008). Migration to the Maya Biosphere Reserve, Guatemala: Why place matters, Human Organization, 67, 37-48.

Casillas, R. (2020). Migración internacional y cambio climático: conexiones y desconexiones entre México y Centroamérica. Revista Latinoamericana de Estudios de Seguridad, (26), 73-92.

Castañeda, G., (24 de marzo de 2020). Re: necesito sus apoyo contestando un breve cuestionario, [correo electrónico] 24 mar. 2020 22:12hrs.

Center for International Environmental Law (CIEL) \& CARE International. (2015). Climate change: tackling the greatest human rights challenge of our time- recommendations for effective action on climate change and human rights. http://www.carefrance.org/ressources/themas/1/4566,CARE_ and_CIEL__Climate_Change_and_.pdf

CIEL \& FES. (2009). Human Rights and climate change. Practical steps for implementation. Switzerland, The Friedrich- Ebert-Stiftung, 34p. http://library.fes. de/pdf-files/bueros/genf/06598.pdf

CMNUCC. (2011). Informe de la Conferencia de las Partes sobre su $16^{\circ}$ período de sesiones, celebrado en Cancún del 29 de noviembre al 10 de diciembre de 2010, Berlín, UNFCCC, 33p. disponible en https://unfccc.int/resource/docs/2010/cop16/ spa/07a01s.pdf
CMNUCC, (2015a). FCCC/CP/2015/L.9 Aprobación del Acuerdo de París, Bonn, UNFCCC. http://unfccc. int/resource/docs/2015/cop21/spa/logs.pdf

CMNUCC. (2015b). FCCC/CP/2015/7, Informe de síntesis sobre el efecto agregado de las contribuciones previstas determinadas a nivel nacional, Bonn, CMNUCC, los dos grados. http://unfccc.int/ resource/docs/2015/cop21/spa/07s.pdf

CNDH. (2018). Protección civil y derechos humanos. Diagnóstico actual de los impactos de los desastres y las estrategias de protección civil sobre la dignidad de las personas. Entrega Final. México CNDH/UNAM-PUEC, 180p. https://www.cndh. org.mx/sites/default/files/documentos/2019-06/ Proteccion-Civil-DH.pdf

Comins Mingol, I. (2016). La Filosofía del Cuidado de la Tierra como Ecosofía en Daimon. Revista Internacional de Filosofía, (67), 133-148 ISSN: 1130-0507 (papel) y 1989-4651 (electrónico). http://dx.doi. org/10.6018/daimon/201501

Comisión Centroamericana de Ambiente y Desarrollo, CCAD. (2010). Estrategia Regional de Cambio Climático. Documento Ejecutivo, El Salvador, Sistema de la Integración Centroamericana

Comisión Centroamericana de Ambiente y Desarrollo, CCAD. (2018). Estrategia Regional de Cambio Climático (ERCC). Actualizada. Plan de Acción 2018-2022, El Salvador, Sistema de la Integración Centroamericana.

Corona, M. Á. (2018). El conocimiento, la percepción y disponibilidad para afrontar el cambio climático en una población emergente, los migrantes de retorno. Estudios Sociales Revista de Alimentación Contemporánea y Desarrollo regional, 28(52). DOI: https://doi.org/10.24836/es.v28i52.578

Corona, M. A. \& Ortiz, B. (2015). Emigración internacional en Puebla bajo condiciones de cambio climático, una perspectiva de análisis diferente. En Ana María Aragonés (Coord.). Consecuencias del cambio climático sobre la migración: un análisis para México, México: UNAM-Instituto de Investigaciones Económicas. pp.135-165. 
Corona, M. A. \& Ortiz, B. (2019). Políticas públicas de desarrollo regional para el cambio climático hacia el 2020 en contextos de marginación y de migración internacional. Estudios Sociales Revista de Alimentación Contemporánea y Desarrollo Regional 29(53). DOI: https://doi.org/10.24836/ es.v29i53.703

Derman, B. B. (2020). Climate Wrongs and Human Rights. In: Struggles for Climate Justice. Palgrave Macmillan, Cham. DOI: https://doi. org/10.1007/978-3-030-27965-3_2

Dryzek, J. (2016). "Institutions for the Anthropocene: Governance in a changing earth system". British Journal of Political Science, 46(4), 937-956.

Duyck, S., Lennon, E., Sherpa, L., Rawe, T., Rosemberg, A., Wessendorf, K., Lund Petersen, L. M., Camacho, M. \& Vélez Uribe, J. (2017). Concretando las Promesas de París: Combatir el Cambio Climático Protegiendo Derechos Recomendaciones para las $\mathrm{Ne}$ gociaciones del Libro de Reglas de París. https://www. ciel.org/wp-content/uploads/2017/09/Delivering-On-Paris-Spanish-Sep2017.pdf

Eckstein, D., Kunzel, V. \& Schafer, L. (2018). Clobal climate risk index 2018 Who Suffers Most From Extreme Weather Events? Weather-related Loss Events in 2016 and 1997 to 2016 https://germanwatch. org/en/download/20432.pdf

El-Hinnawi, E. (1985). Environmental Refugees. Nairobi. Programa de Naciones Unidas para el Medio Ambiente.

Farbotko, C. \& Lazrus, H. (2012). The first climate refugees? Contesting global narratives of climate change in Tuvalu. Clobal Environmental Change, 22(2), 382-90. DOI: https://doi.org/10.1016/j. gloenvcha.2011.11.014

de Loma-Osorio, G. F. y. (2016). The 2030 Agenda for Sustainable Development: Bringing Climate Justice to Climate Action. Development 59, 223-228. https://doi.org/10.1057/s41301-017-0122-9

Gardiner, S. (2011). A Perfect Moral Storm, Oxford, New York.

Glemarec, Y. (2019). Aligning National Interests and Clobal Climate Justice: The Role of Human Rights in Enhancing the Ambition of Nationally Determined Contributions to Combat Climate Change. Fudan ]. Hum. Soc. Sci. 12, 309-327. https:// doi.org/10.1007/s40647-018-0249-4
González, H., Salazar, J. \& Cruz, R. (2012). Contaminación como uno de los determinantes de la migración. Evidencia para México. Revista Economía Mexicana, nueva época. XXI(1), 69-92.

Gössling, S. \& Humpe, A. (2020). The global scale, distribution and growth of aviation: Implications for climate change. Clobal Environmental Change, 65, 102194. https://doi.org/10.1016/j.gloenvcha.2020.102194

Griffin, P. (2017). The Carbon Majors Database CDP Carbon Majors Report 2017100 fossil fuel producers and nearly 1 trillion tonnes of greenhouse gas emissions https://b8f65cb373b1b7b15feb-c7od8ead6ced550b4d987d7co3fcdd1d.ssl.cf3.rackcdn.com/ cms/reports/documents/000/002/327/original/ Carbon-Majors-Report-2017.pdf?1499691240

HAC. (Dec 14, 2018). Statement On Stepping Up Climate Ambition. [The Statement On Stepping Up Climate Ambition remains open for countries and non-party stakeholders to support. Join us]. [tuit]. https://twitter.com/HACoalition/status/1073682492962603008

Hák, T., Svatava, J. \& Moldan, B. (2015). Sustainable Development Coals: A need for relevant indicators, Ecological Indicators, 60, 565-573, DOI: 10.1016/j.ecolind.2015.08.003

Helm, D. (2016). "The future of fossil fuels-is it the end?". Oxford Review of Economic Policy, 32(2), 191205.

Hou, J., Walsh, P. P., \& Zhang, J. (2015). The dynamics of Human Development Index, The Social Science Journal, 52, 331-347. http://dx.doi.org/10.1016/j. soscij.2014.07.003

Hsiang, S. M. \& Sobel, A. H. (2016). Potentially Extreme Population Displacement and Concentration in the Tropics Under Non-Extreme Warming. Scientific Reports, 6, 25697. https://doi. org/10.1038/srep25697.

IPCC. (2013). Cambio climático 2013 Bases físicas. Resumen para responsables de políticas Informe del Crupo de trabajo I del IPCC, IPCC, Ginebra, Suiza. http://www.ipcc.ch/pdf/assessment-report/ar5/ wg1/WG1AR5_SummaryVolume_FINAL_SPANISH.pdf 
IPCC. (2014a). Cambio Climático 2014. Mitigación del cambio climático. Resumen para responsables de políticas, IPCC, Ginebra, Suiza, 33p. http:// www.ipcc.ch/pdf/assessment-report/ar5/wg3/ WG3AR5_SPM_brochure_es.pdf

IPCC. (2014b). Climate change 2014. Synthesis Report. Contribution of Workings Groups I, II and III to the Fifth Assessment Report of the Intergovernmental Panel on Climate Change. Geneve, IPCC, p.151.

IPCC. (2019). Clobal Warming of $1.5^{\circ} \mathrm{C}$. An IPCC Special Report on the impacts of global warming of $1.5^{\circ} \mathrm{C}$ above pre-industrial levels and related global greenhouse gas emission pathways, in the context of strengthening the global response to the threat of climate change, sustainable development, and efforts to eradicate poverty, IPCC, Ginebra, 616p. https://www.ipcc.ch/site/assets/uploads/ sites/2/2019/06/SR15_Full_Report_Low_Res.pdf

Ivanova D, Wood, R. (2020).The unequal distribution of household carbon footprints in Europe and its link to sustainability. Clobal Sustainability, 3(e18), 1-12. https://doi.org/10.1017/sus.2020.12

Kahane, A. (2018). Colaborar con el enemigo. Cómo trabajar con quien no estás de acuerdo, no te agrada o no confías. México, UNAM-CNDH, 192p.

Keohane, R. O. \& Oppenheimer, M. (2016). Paris: beyond the climate dead end through pledge and review? Harvard Project on Climate Agreements, Discussion Paper 2016-85, Cambridge.

Ki-Moon, B. (2009). Stressing Need for Rapid Action at World Climate Conference, Secretary-Ceneral Says Anti-Science, Economic Scaremongering Could Lead to Widespread Disaster, NY, United Nations, Meeting's coverage and press release https://www.un.org/press/en/2009/sgsm12433. doc.htm

Kirsch, A., Opeña Disterhoft, ]., Marr, G., McCull, P., Gürsöz, A., Aitken, G., Hamlett, C., Saldamando, A., Louvel, Y., Pinson, L., Cushing, B., Brown, C. \& Stockman, L. (2020). Banking on Climate Change Fossil Fuel Finance Report 2020, California, Estados Unidos, Rainforest Action Network, Bank Track, Indigenous Environmental Network, Oil Change International, Reclaim Finance and Sierra Club, 138p. https://www.ran.org/wp-content/ uploads/2020/03/Banking_on_Climate_Change_2020_vF.pdf
Koivurova, T., Duyck, S. \& Heinämäki, L. (2013). Climate Change and Human Rights In Erkki ]., Hollo, Kati Kulovesi. \& Michael Mehling (Eds.). Climate Change and the Law, , lus Centium: Comparative Perspectives on Law and Justice Volume 21 Dordrecht: Springer, pp. 287-325. https://www.arcticcentre.org/loader.aspx?id=6ca1e266-08a3-4459-aoac-3834bc7ba4be

Krämer, L. (2019). Climate Change, Human Rights and Access to Justice. Journal for European Enviromental \& Planning Law, 16, 21-34 https://doi. org/10.1163/18760104-01601003

Kumari Rigaud, K., de Sherbinin, A., Jones, B., Bergmann, J., Clement, V., Ober, K., Schewe, J., Adamo, S., McCusker, B., Heuser, S., \& Midgley, A. (2018). Croundswell: Preparing for Internal Climate Migration. Washington, DC: The World Bank. http://documents.worldbank.org/curated/ en/846391522306665751/pdf/124719-v2-PUBPUBLIC-docdate-3-18-18WBG-ClimateChangeFinal.pdf

Lejtreger, R. (2018). La movilidad humana en la agenda climática de las Américas. Necesidades y Oportunidades. San José, Costa Rica; Centro Regional de Colaboración/CMNUCC- Organización Internacional para las Migraciones/Oficina Regional para Centroamérica, Norteamérica y el Caribe

Lonergan, S. \& Swain, A. (1999). Environmental Degradation and population displacement. Aviso, 2, 1-11.

López Morales, L. (2019). La educación climática como una medida de adaptación al cambio climático. En Ibarra Sarlat, Rosalía (Coord.). Cambio climático y gobernanza. Una visión transdisciplinaria, (pp.175-196). UNAM/Instituto de Investigaciones Jurídicas, México.

Lozano Sivisaca, D. C., Chacón-Cascante, A., Cutiérrez Montes, I. \& Robalino, J. (2015). Eventos climáticos extremos y migración interna en Cuatemala, un análisis basado en percepciones de expertos. CIENCIA ergo-sum, 22(1), 35-44.

Mancini, F. (2018). El riesgo en la sociedad contemporánea. En Rubio Carriquiriborde, Ignacio (Coord.). Sociología del riesgo. Marcos y aplicaciones. (pp. 17-48). UNAM/ Facultas de Ciencias Políticas y Sociales, México, 242p. 
Meadowcroft, ]. (2009). "What about the politics? Sustainable development, transition management, and long term Energy transitions". Policy Science, 42, 323-340.

Methmann, C. \& Oels, A. (2015). From "fearing" to "empowering" climate refugees: Governing climate-induced migration in the name of resilience. Security Dialogue 46(1), 51-68. DOI: https:// doi.org/10.1177/0967010614552548

Narváez, L., Lavell, A. \& Pérez Ortega, C. (2009). La gestión del riesgo de desastres: un enfoque basado en procesos. Lima, Perú. Proyecto Apoyo a la Prevención de Desastres en la Comunidad Andina PREDECAN, Comité Andino para la Prevención y Atención de Desastres-CAPRADE

Nawrotzki, R., Riosmena, F., \& Hunter, L. (2013). Do rainfall deficits predict U.S.-Bound migration from rural Mexico? Evidence from the mexican census. Population Research Policy Review, 32, 129-158.

Nordhaus, W. (2014). The Climate Casino, Yale, New Haven.

Nordhaus, W. (2015). "Climate clubs: Overcoming free-riding in international climate policy". American Economic Review, 105(4), 1339-1370.

Ochoa Lupián, L. E., \& Ayvar, F. J. (2015). Migración y cambio climático en México, CIMEXUS, X(1), 35-51

OHCHR. (2020). Human Rights Council resolutions on human rights and climate change. https://www. ohchr.org/EN/Issues/HRAndClimateChange/ Pages/Resolutions.aspx

Organización Internacional para las Migraciones, OIM. (2019). Informe sobre las Migraciones en el Mundo 2020. OIM, Ginebra, Suiza. https://publications.iom.int/system/files/pdf/wmr_2020_ es.pdf

Ostrom, E. (2009). "A polycentric approach for coping with Climate change". Policy Research Working Paper 5095

Oxford University Press. (2019). Oxford Dictionaries, Word of the year 2019, UK, Oxford University Press, https://languages.oup.com/word-of-theyear/word-of-the-year-2019

Paavola, ]. (2007). "Institutions and environmental governance: a reconceptualization”, Ecological Economics, 63, 93-103
Paavola, J. (2008). Explaining Multi-Level Environmental Covernance, Sustainability Research Institute, University of Leeds.

Pearson, L. \& Pelling, M. (2015). The UN Sendai Framework for Disaster Risk Reduction 20152030: Negotiation Process and Prospects for Science and Practice. World Scientific Publishing Company ] Extreme Events, 2(1), 1571001 DOI: https:// doi.org/10.1142/S2345737615710013

PNUMA. (2019). Informe sobre la disparidad en las emisiones de 2019. Programa de las Naciones Unidas para el Medio Ambiente, Nairobi. https://wedocs. unep.org/bitstream/handle/20.500.11822/30798/ ECR19ESSP.pdf? sequence $=17$

Trogrlić, R., Cumiskey, L., Triyanti, A., Duncan, M. J., Eltinay, N., Hogeboom, R. J., Jasuja, M., Meechaiya, C., Pickering, C. J., \& Murray, V. (2017). Science and Technology Networks: A Helping Hand to Boost Implementation of the Sendai Framework for Disaster Risk Reduction 2015-2030? International journal of disaster risk science (IJDRS), 8(1), 100-105. https://doi.org/10.1007/s13753-0170117-X

Sánchez Rodríguez, R., Ürge-Vorsatz, D. \& Barau, A.S. (2018). Sustainable Development Coals and climate change adaptation in cities. Nature Clim Change, 8, 181-183. https://doi.org/10.1038/ s41558-018-0098-9

Schapper, A. \& Lederer, M. (2014). Introduction: Human rights and climate change: mapping institutional inter-linkages. Cambridge Review of International Affairs, 27(4), 666-679. DOI: https://doi.org/ 10.1080/09557571.2014.961806

Schwarz-Herion, O. (2018). The Impact of the Climate Change Discussion on Society, Science, Culture, and Politics: From The Limits to Growth via the Paris Agreement to a Binding Clobal Policy? In: Omran, Abdelnaser \& Schwarz-Herion, Odile (Eds). The Impact of Climate Change on Our Life. The Questions of Sustainability. (pp. 1-32). Springer Nature Singapore, Gateway East, Singapore. https:// doi.org/10.1007/978-981-10-7748-7

Sen, A. (2010). La idea de la justicia. México, Taurus, 499p. 
Sepúlveda Pizarro, ]. (2018), Ecosofía: hacia una comprensión de la sabiduría de la tierra desde la noción de ritmo del ser de Raimon Panikkar. lu. Revista de Ciencias de las Religiones, 23, 263-278.

Stavins, R. N. (2016a). The Paris Agreement lays a good foundation for Climate progress. The Environmental Forum, 33(3), 13

Stavins, R. N. (2016b). The Paris Agreement-A good foundation for meaningful progress. Green Fiscal Policy Network Newsletter, 4, 1-4.

Tamayo Pérez, L. (2017). La locura ecocida. Ecosofía psicoanalítica. México, Fontamara, Colección Argumentos, 165p.

The Guardian. (2017). Just 100 companies responsible for $71 \%$ of global emissions, study says, The Cuardian. https://www.theguardian.com/sustainable-business/2017/jul/10/100-fossil-fuel-companies-investors-responsible-71-global-emissions-cdp-study-climate-change

Tol, R. (2015). "The road form Paris: international climate policy after the Paris agreement of 2015", Vox, CEPR,s Policy Portal. http://voxeu.org/article/ road-cop21

Toussaint, P. \& Martínez, A. (2019). A human rightsbased approach to loss and damage under the climate change regime. Climate Policy, 20(6), 743757. DOI: https://doi.org/10.1080/14693062.2019. 1630354

UNEP \& Columbia Law School. (2015). Climate Change and Human Rights. Nairobi, Nenya, United Nations Environment Programme, 43p. https:// web.law.columbia.edu/sites/default/files/microsites/climate-change/climate_change_and_human_rights.pdf

Unesco. (2017). Actas de la Conferencia General $39^{\mathrm{a}}$ reunión París, 30 de octubre - 14 de noviembre de 2017, Volumen 1 Resoluciones, Anexo III Declaración de Principios Éticos en relación con el Cambio Climático, París, Organización de las Naciones Unidas para la Educación, la Ciencia y la Cultura, 188p. pp. 161-167. https://unesdoc.unesco.org/ark:/48223/pfooo0260889_spa

Vega, H. (2005). Migración ambiental inducida por variabilidad climática: El caso del Corredor Centroamericano de la Sequía. San José, Costa Rica, CEMEDE.
Weichselgartner, J. \& Pigeon, P. (2015). The Role of Knowledge in Disaster Risk Reduction. Int $]$ Disaster Risk Sci, 6, 107-116. https://doi.org/10.1007/ s13753-015-0052-7

WMO. (2016). El Estado Mundial del Clima 2011-2015, Ginebra, WMO.

WMO. (2019). The Clobal Climate in 2015-2019, WMO, Ginebra, 21p. https://library.wmo.int/doc num.php?explnum_id=9936

World Economic Forum, WEF. (2019). The Clobal Risks Report 2018, 14th Edition, World Economic Forum. Geneva, Switzerland, 114p. http://www3. weforum.org/docs/WEF_Global_Risks_Report_2019.pdf

Young, O. R. (2013). On Environmental Covernance, Paradigm Publishers, London 